\title{
Focus on Clinical Best Practices, Patient Safety and Operational Efficiency
}

Brenda Laurie-Shaw, Wendy Taylor and Carol Roach

\section{Introduction}

The following article explores the MOE/MAR-driven changes from the perspective of nurses. The examination of the collaboration and coordination of the Nursing Informatics (NI) Team with Nursing, the role of Nursing Informatics, the collaboration with the Project Team from Shared Information Management Services (SIMS), the Education Working Group and the Computer User Support Program (CUSP) are features of this journey into the electronic world during the implementation of MOE/MAR.

\section{Background}

The nursing staff at UHN represents the largest group of employees and clinicians at UHN. Prior to the implementation of Medication Order Entry/Medication Administration Record (MOE/MAR), nursing staff used the HIS for processing patient admission assessments, entering written orders for diets and reviewing laboratory and diagnostic test orders and results and patient chart content. Despite a large portion of the patients' results being available electronically, almost no electronic clinical documentation had been implemented, creating the need for all clinicians to work in a hybrid paper-electronic environment.

At UHN, it was decided that MOE/MAR would be implemented before electronic clinical documentation because of the significant impact it would have on patient safety. This approach had pros and cons. It was generally felt that on-line medication order entry would have a positive impact on all other order entry. A downside of this approach, albeit only in the short term, was that MOE/MAR necessitated greater adoption of the HIS by nurses, becoming a mandatory medication management tool used by all nurses on a continuous basis. One can only speculate that had clinical documentation been implemented prior to MOE/MAR, nurses might have been more comfortable using computers by the time MOE/MAR was implemented. As a result, the need for significant change management to ensure $100 \%$ adoption by Nursing was critical for success.

\section{Change Management}

Moving from a paper-based medication management environment to completely on-line processes was a major change for nurses. The key to a successful transition for nurses was to have Nursing Informatics be an integral part of the Project Team to facilitate the following:

- understanding of nursing workflow

- education of nurses about the MOE/MAR vision and project management process

- preparation of nurses to successfully manage the change

- identification, documentation and resolution of challenges affecting workflow, workload and policies

- support for nurses' ongoing learning and problem-resolution processes for post-implementation issues 
A key aspect of the project was to address the concerns of nurses about becoming less efficient; as experts in the field of medication management, nurses were concerned that the move from paper to electronic could result in efficiency and competency challenges, while they were becoming proficient with the technology. Important confidence-building tactics were part of the change management approach and included efforts to involve nurses in the design of the MOE/MAR, answer nurses' questions promptly and review the process for solving go-live problems at daily meetings.

\section{Nursing Influence on Design}

The consultative role of Nursing Informatics was the primary way in which the needs of nurses were incorporated into the design of MOE/MAR. Nursing Informatics comprised a diverse group of experienced nurses who combined their clinical knowledge with an understanding of the information requirements of nurses and the use of technology in the nursing environment. Nursing Informatics is a corporate Nursing department reporting through a Director to the Vice-President and Chief Nurse Executive. In addition to the Director of Nursing Informatics, the department also included four Project Managers, eight Clinical Support Analysts (described later in this article) and an Analyst. The role of Nursing Informatics is to identify nursing information and knowledge requirements to ensure they are accommodated by new technologies and, thus, effectively incorporated into nursing practice.

For the MOE/MAR project, Nursing Informatics acted as "translator," spearheading the gathering of nursing input and making nurses' requirements known to the SIMS Project Team. To maintain consistency across the enterprise, one Nursing Informatics Project Manager was dedicated full time to MOE/ MAR; a second full-time resource led each of the individual clusters.

As part of the MOE/MAR project, the specific roles of the Nursing Informatics resources included

- assessing and documenting nursing workflow

- collaborating with Pharmacy and SIMS to establish technical development standards for electronic orders, incorporating available best practice and safety guidelines information

- configuring the customizable parts of the system with the SIMS technical development team

- collaborating with SIMS on screen design

- interpreting and clarifying pre-printed orders to the SIMS technical development team

- testing new development

- conducting user trials for device selection

- recruiting and training clinical support personnel

- assisting with curriculum design for nursing training
- resolving nursing workflow and other process issues during implementation (e.g., the role of transient agency nurses in workflow)

- addressing clinical policy issues that arose as a consequence of MOE/MAR

Not only did Nursing Informatics' input lead to a better design, but having Nursing working with SIMS as "co-creators" was also considered a wise political decision by the Project Team and the hospital senior management.

During the ramp-up to the MOE/MAR pilot project, and then again as part of the go-live preparations for each hospital service implementation (referred to as "clusters"), Nursing Informatics worked with nurse managers, clinical educators and practising nurses to create the workflows for that service. Medical Informatics, Pharmacy Informatics and Nursing Informatics spent considerable time negotiating solutions required to resolve workflow challenges during the pilot and first cluster. This was done through an ongoing series of meetings on the ward to discuss system requirements and also by observing and documenting their daily medication-related activities. This information was then used to map the nurses' existing paper-based medication management workflow. Work redesign programs often highlighted new, changed or different workflow requirements to reduce potential patient safety risks. These mappings covered the typical drug management processes - notification, verification, preparation, administration and documentation.

\section{Order Notification}

Nursing needs uniquely shaped the design of the MOE/MAR environment at UHN. The advent of the "electronic whiteboard" order notification tool is illustrative of how this took place. Previously with the paper medical record, a chart flag system was used to inform nurses of new orders. Nurses required a similar notification system in the hybrid paper/electronic environment. Even with on-line medication management, Nursing Informatics felt that nurses would not be logged into the system often enough to use the Inbox functionality within the system. In order to reduce the risk of missed electronic orders, the electronic whiteboard notification tool was created to mimic the whiteboard typically found in a nursing station. An electronic whiteboard would help make the transition to MOE/MAR more familiar for nurses by making electronic orders visible without logging in. In this case, this notification tool was a dedicated computer monitor at each nursing station used to notify nurses of new orders requiring their attention (see Figure 1).

Although Nursing's adoption of the electronic whiteboard varies from hospital service to hospital service, it is widely felt to have improved the overall transition to dealing with electronic orders. 
Figure 1. UHN testing inpatient unit whiteboard

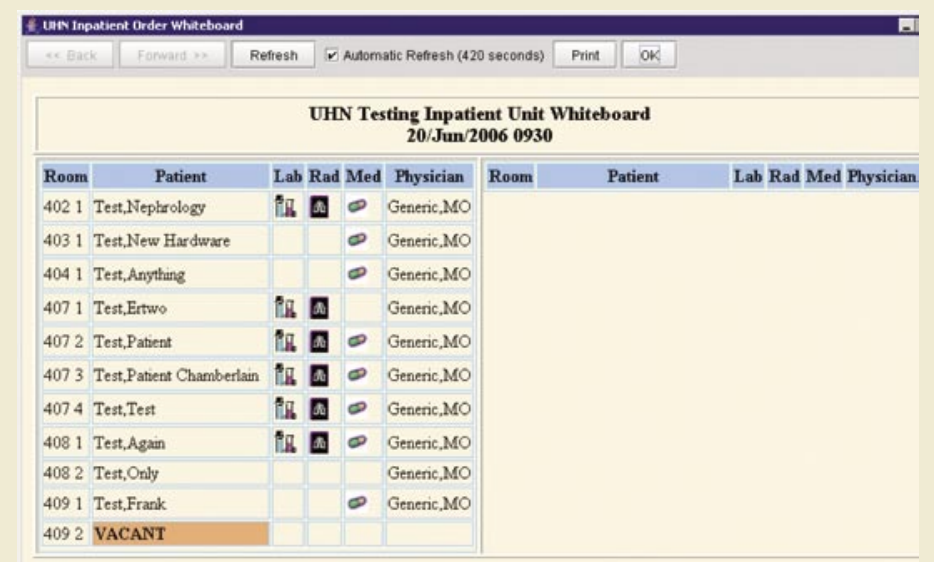

tion, security and sufficient storage capacity for multiple patients' medications and other nursing tools taken to the bedside. Although none of the devices evaluated met all of Nursing's and SIMS's criteria for an "ideal" device (devices favoured by nurses were unaffordable in large numbers), a decision was eventually made to go with a mobile wireless computer on carts.

The mobile devices on carts did yield immediate patient safety improvements by eliminating transcription and by increasing nurses' ability to correctly read the orders. However, the patient identification process was disrupted in relation to safe medication practice with the use of the carts. Since the carts were too large to be taken into many patient rooms, they were often left in the hallways. The carts were also found to be slower than the speed at which an unencumbered nurse wishes to move. Because the carts (which could not always accommodate the medications and other supplies nurses needed at the bedside)

\section{Verification and Preparation}

Nurses contributed to the design aspect of the project. For example, nurses determined that to support medication preparation, the MOE/MAR application should include a link to an online drug reference manual and recent relevant lab values. These links were easily incorporated and assisted with nurse adoption. Another design contribution by Nursing was to develop reports that improved data display within the electronic system. A "Med Prep Report" tool was created to replace the paper MAR in providing information for medication preparation. This report assembled information such as the medication order, last dose, last administration site (for injectables) and special instructions from the physician, pharmacist, another nurse and the patient. Nurses also wanted a display of the seven-day history of medication administration, which was also accommodated with the development of an electronic report. The physician group found this report valuable as well to support communications with nurses.

The workflow analysis showed that nurses needed to have easy access to the MOE/MAR system at all "points of activity" during the medication management process. As part of the MOE/MAR deployment, a computer was installed on an articulating arm beside each drug dispensing machine. This gave nurses access to up-to-date patient and MAR information.

\section{Administration and Documentation}

The shaping of the MOE/MAR workflow around nurses' needs continued to the medication administration and documentation stages, most visibly in the form of the nurses' mobile medication carts. One of the greatest challenges was finding an appropriate mobile solution that combined a computer with transporta- were too large to move into a patient room, nurses sometimes eliminated the direct verification of the MAR with the patient. Not performing this last step of the "Five Rights" at the bedside hampered positive patient identification. Although the former paper-based MAR was also not always taken into a patient room prior to MOE/MAR to ensure positive patient identification, the carts made this step even more difficult. The design and utility of these carts remain a challenge that is still being addressed.

It should be noted that UHN chose not to use handheld computer solutions for MOE/MAR. Not only did these not meet the needs of storage and drug transporting, but the graphical user interfaces of existing products available did not meet usability requirements due to their small form-factor.

\section{Adoption Readiness}

Early on, Nursing Informatics observed that a change of the magnitude of MOE/MAR would require a gradual introduction of manageable information chunks to nurses. Developed in parallel with the design of the actual electronic system, a MOE/ MAR training program for nurses was created. An Education Working Group consisting of representatives from Education, the Project Team, Nursing Informatics and CUSP designed and refined the curriculum with each cluster go-live. One particularly effective strategy was to target and incorporate clinically accurate workflow scenarios and examples into the classroom training. A five-step program evolved over several implementations when it was realized that a single, four-hour classroom session was insufficient to prepare such a large group for this major change. These steps included 
- computer skills self-assessment and review by CUSP

- pre-class visit to unit to demo the MAR and answer questions about the upcoming change

- four-hour hands-on MOE/MAR functionality class

- post-class review (hands-on) prior to go-live

- post-go-live functionality review and self-assessment by CUSP

The first step of the program focused on improving basic computer skills through individual coaching by CUSP. In addition, the Nursing Units were visited during the day and the evening by Nursing Informatics and members of the Project Team, who provided previews of the new MAR and addressed nurses' questions. Nurses who had seen the MAR more than once outside the classroom were better prepared to deal with the "hands-on" portion of the learning in the classroom. Those who came in for a four-hour classroom session on their day off were far more comfortable with the learning than those who came after a 12-hour nightshift or during their regular workday. (This was paid time for the nursing staff provided for by their employment contract.)

During the final ramp-up to go-live for each cluster,

Nursing Informatics and the Project Team again visited the Nursing Units to conduct small group "hands-on" refresher sessions to help nurses retain what they had learned in the classroom and to answer any outstanding questions. One of the useful tools was the self-assessment checklist already in use for other skill development programs. A competency assessment tool was created for MOE/MAR based on the required skills for independent functioning at the end of the four-week support period. The resulting checklist, administered by the computer support teachers about two months after the go-live, helped to ameliorate skill gaps. The checklist also identified areas where additional tutoring or coaching would be helpful.

The purpose of the tool was to ensure that nurses learned everything required of them in order to be effective when working with MOE/MAR, rather than serving as a performance review tool for Management. According to the Director of Nursing Informatics, "It also allowed us to focus our training efforts on specific needs rather than putting everyone back into the classroom again." In fact, teaching nurses the "mechanics" of using MOE/MAR was relatively easy compared to preparing them for the extent of the practice and workflow changes they would face.

\section{Engagement}

In the same way that knowledge levels around the use of computers varied widely across Nursing at the beginning of the MOE/MAR project, so did the comfort levels. There were nurses who were already making significant use of online applications and information as part of their everyday workflow and were quite comfortable doing so. Until MOE/MAR arrived, however, there were other nurses who had managed to avoid any significant use of computers through various workarounds.

... teaching nurses the

"mechanics" of using MOE/MAR was relatively easy compared to preparing them for the extent of the practice and workflow changes they would face.

\section{Pilot}

The choice to pilot MOE/MAR in General Internal Medicine (GIM) was particularly appropriate from a nursing perspective. Strong nursing leadership - found in GIM - was important to ensure successful engagement with nurses on that unit. The GIM nurses embraced the opportunity to be pioneers and to help shape the future MAR and medication workflow. User interfaces were tested on this group of nurses, and their workflow needs related to devices were studied in detail. The nurses' concerns challenged the Project Team and Nursing Informatics to find solutions to long-standing problems (e.g., verbal orders). Verbal order practices were one of the most challenging issues throughout whole implementation. Nursing Informatics and a physician champion worked out mutually accepted guidelines for the acceptability of verbal orders. 


\section{First Go-Live}

Although they had been included in all the pre-pilot and golive ramp-up meetings, nurses in the first few units to go-live felt as if they were operating in an entirely new world when it came to using MOE/MAR. Several nurses doubted that physicians would change the way they would do drug order entry, and some nurses were concerned that MOE/MAR would be stopped and all their efforts wasted, should physicians not support MOE/MAR. Consequently, the first go-live after the pilot was Medication Order Entry (MOE) - which ensured that physicians would adopt the system. In retrospect, we underestimated the impact of implementing MOE without MAR. In fact, nurses welcomed the MAR six months later.

During every implementation there were daily meetings with the clinical stakeholders - Nursing Informatics and the Project Team - to identify and resolve issues as they arose. The daily meeting typically reviewed and sought solutions to device, technical, interdisciplinary and training issues. This rapid resolution process contributed significantly to building the confidence of the nurses.

\section{General Rollout}

One of the tactics for planning the rollout was the thoughtful sequencing of clusters; they were organized by service in order of complexity (from least to most, in order to provide a rigorous test of MOE/MAR). As the rollout progressed, nurses in the early clusters became informal "ambassadors." Through discussions with their peers, they were able to help nurses in other units understand MOE/MAR better as well as gain honest insight into benefits and challenges of MOE/MAR. Although nurses were invited to visit already-live units, few availed themselves of this opportunity. More effective was the distribution and discussion (by nurses with nurses) of a series of photos depicting "a day in the life" of a MOE/MAR nurse.

Another positive dynamic that did occur among nurses adapting to MOE/MAR was the harmonizing effect MOE/ MAR had on their units. An attitude along the lines of "we're all in this together, so let's work together to make it happen" emerged. On the other hand, MOE/MAR tended to reveal the best and worst of the clinical practices within the unit. This was valuable as it uncovered underlying clinical and process changes required at UHN.

\section{Patterns of Adaptation}

Over the course of the rollout, Nursing Informatics observed a consistent pattern in the reaction by nurses using MOE/MAR. There was intense scrambling during the week before go-live - Day 1 was chaotic and Day 2 was very tough. But noticeable improvement was made as early as the week's end. Indeed, the nurses were surprised at how quickly they saw the potential value in the system and how quickly they adopted it.
It also turned out that it was newer nurses - not the older ones as many had expected - who had the most trouble learning to use MOE/MAR. This was primarily due to the fact that they were struggling to learn nursing practices at the same time as learning how to use a new system.

\section{One of the tactics for planning the rollout was the thoughtful sequencing of clusters; they were organized by service in order of complexity.}

The Clinical Support Analysts (CSA) provided 24/7 support to clinicians for four weeks following the go-live, and played a major role in helping nurses become comfortable using MOE/ MAR. CSAs are nurses with informatics training skills. The big change and the big disruption that comes with implementing a capability such as MOE/MAR could easily shake any nurse's self-confidence and judgment; having the CSAs close at hand was very reassuring. With these knowledgeable peers readily available to them to address questions and concerns, nurses were more inclined to begin using the system. On average, nurses were fully up to speed using the system after only two weeks.

\section{Shared Accountability}

As expected with any initiative of this size and complexity, a wide variety of challenges were encountered throughout the MOE/ MAR rollout. Clinical transformation was viewed as a collaborative effort with all the stakeholders who worked together in an iterative process and which resulted in better communication and acceptance of policy decisions.

For example, during the initial go-live periods, nurses were concerned about how they would be able to maintain a high level of patient care at the same time as they were trying to learn to use MOE/MAR. To help nurses with their workload during this period, the project did provide limited funds in the early clusters to allow for some additional nursing staff. Each nurse would be responsible for fewer patients, thus giving them more time to work on learning the new system. Due to insufficient project funding, however, it was not possible to provide this same support across all the clusters going live.

MOE/MAR also affected UHN policies related to nursing practice. Prior to MOE/MAR, UHN had a policy that medications were to be administered within 30 minutes before or after the scheduled time. It was never known whether this actually occurred. UHN moved to a generous two-hour window before and after the scheduled time on the electronic MAR; it was 
recognized that the 30-minute administration time window was unachievable while nurses were learning. The two-hour policy still remains, as there have been no further administration issues with the change. However, UHN will continue to assess the opportunity to reduce this window.

Although the list of technical challenges had been considerably reduced from those identified during the pilot, there were still many design problems and workarounds that continued to frustrate nurses. Some common drugs still cannot be ordered on-line, due to the complexity of the protocols associated with them (e.g., insulin and heparin drips). Some orders must be handwritten; some documentation is still manual, thus perpetuating a hybrid paper-electronic environment (which nurses find frustrating and leads to extra work). Nursing Informatics and SIMS continue to work together with the hospital information system vendor to address these and other ongoing issues.

\section{Lessons Learned}

1. Personnel with first-hand nursing experience need to ensure that nurses' requirements are incorporated into system design and workflow specifications.

2. An effective and consistent support strategy is important. Ensure that support personnel appreciate clinical needs, are visible and accessible, and have both clinical and technical expertise.

3. Computer knowledge and comfort levels will vary widely. Bring the levels of computer skills as close to one level as possible before MOE/MAR training.

4. If possible, project planning and budgeting should include a provision for adding temporary additional nurses to any service during the go-live period to relieve workload concerns. This will give the permanent full-time nurses a chance to learn the new system.

5. Understand and assess necessary competencies in order to identify required training.

6. Ensure that the organization's helpdesk support model is adequate to support a newly operational MOE/MAR Unit; personnel who can distinguish between technical and clinical/ practice challenges or problems are important.

7. Recognize that such significant change will make nurses feel like novices until they are able to re-establish the workflow and patterns that previously made them efficient.

8. Gaps and inconsistencies in the practice and workflow between hospital services will be revealed by the implementation of MOE/MAR. They should be resolved and standardized as much as possible.

9. Expect that there will be at least one workflow or practice surprise on go-live day. Be prepared to resolve it quickly.

10. Incident reporting may increase significantly when MOE/MAR is first implemented due to access to better information.

\section{Sustaining Change}

Although the go-live point for each cluster was clearly a major milestone for the MOE/MAR project, it was not the end. As they ramped up to full speed with MOE/MAR, nurses needed continuous support to reinforce their training and increase their confidence. For the first four weeks following the go-live, this support was provided by the CSAs and Project Team members stationed on the ward (available to nurses on a $24 / 7$ basis). In the later clusters, red lab coats worn by the support team members made them even more visible to clinicians.

The CSAs were initially comprised of nurses recruited from throughout the hospital to join the support team on a temporary basis. Due to the general shortage of nurses, the borrowed staff had to return to their home units after each go-live support period, and a new group of seconded nurses were brought in to provide support. Initially, there were no nurses who were expert in the system, except in Nursing Informatics. This was problematic because there was a long learning period to master the system. The project would lose the benefit of the continuous knowledge-building from one cluster to the next.

As a result, Nursing Informatics and SIMS created a business case for a more permanent team of CSAs and went to the Occupational Health department to determine who might be available from the pool of nurses that were injured or otherwise unable to provide direct care. The CSA team also included some nurses who were interested in exploring Informatics as a career option.

By all measures, it appeared nurses were very positive about the CSA concept. They trusted the CSAs and were willing to approach them if they were having difficulty or if they wanted to discuss a practice issue without feeling threatened. The way that nurses engaged with the CSAs was different than the way they worked with the SIMS group - it was a nurse-to-nurse relationship and it appears that nurses felt more comfortable discussing the technical functionality in this context.

Deployments were made quickly to get the hospital up and running on MOE/MAR as soon as possible to minimize the hybrid environment. However, a backlog of "unfinished business" was left following the go-live. As a result, CSAs would circle back to answer the more advanced questions that arose; to investigate data that were puzzling to nurses; to assess whether there were any deviations from the prescribed workflow that could potentially affect data quality or safety; and to conduct audits that would verify that the system was being used safely. Audits performed by the CSAs after cluster implementations monitored several key aspects, including

- allergy documentation that used free text or outdated information

- duplicate orders in the system, which pose a risk for doubledosing the patient 
- medication orders that were inappropriately written on paper and risked being missed

- doses of medication that were not documented or cancelled inappropriately

CSAs continue to conduct these audits.

In addition, UHN established a unique process with its helpdesk vendor to support MOE/MAR. Nurses originally found they could not get much help through the helpdesk because the vendor personnel did not have the required understanding of MOE/MAR's complexities. To improve the level of ongoing MOE/MAR support, SIMS worked with the helpdesk to establish a new "MOE/MAR option" on its helpdesk phone system that would automatically route the call to support staff specifically trained for that purpose.

\section{Creating Safer Care Processes}

The implementation of MOE/MAR revealed differences from cluster to cluster and from service to service in many clinical workflows. More importantly, it also revealed inconsistencies and gaps in procedures and policies. Although few, if any, of these problems were actually caused by MOE/MAR, a comprehensive project such as this had the effect of putting existing clinical practices under the microscope.

An example is seen in how UHN addressed incident reporting. Incident reporting was not comprehensively done at UHN prior to MOE/MAR because of the fear of blame. During the four-week go-live support period, Nursing Informatics and CSA staff spoke with nurses about the importance of reporting incidents, helped them write the reports and investigated the causes of the incidents to see if they had anything to do with MOE/MAR. Their goal was to get nurses to start seeing incident reporting as a learning mechanism rather than as punitive. With the growing focus on patient-centred care at UHN, the reporting of medication incidents has become an even more important business metric. The MOE/MAR project offered an opportunity to change perceptions around incident reporting - truly, a blame-free culture focused on patient safety.

A more significant challenge arose regarding UHN's Acute Pain Service (APS). In pre-MOE/MAR days, a nurse working in any unit could receive two drug orders for the same patient - one from a physician on the ward and the other from an APS physician, both of whom might believe their order had the highest priority. The nurse typically ended up having to sort this out, often requiring considerable discussion with the physicians. The absence of a pre-defined and consistent process in this area was brought to light when the Project Team attempted to analyze and map the APS workflow into MOE/MAR. It was also discovered that there were different practices at two of UHN's hospitals, and MOE/MAR had to be configured in a way that brought these two together into a single, consistent workflow. The Director of Nursing Informatics noted: "We were amazed at the lack of standardization of clinical practices across the organization and the challenges of bringing it all together."

\section{Conclusion}

Even though the technology behind MOE/MAR continues to evolve, MOE/MAR in its early days has proven profoundly valuable to nurses. Medication information is clearer, and thus there are fewer transcription errors. The previous need to copy order information from an expired paper MAR to a new paper MAR has been eliminated. Patient medication information is available more quickly (and in general, all the information is confined to one place). And nurses can easily obtain different views of this information.

Nevertheless, some inefficiency remains. Following new workflows or policies requires learning. Transforming electronic tasks from manual ones may require some nurses to spend more time than they did previously following up with physicians to clarify orders or other questions. In other cases, following good practices simply takes longer. Either way, MOE/MAR is rapidly becoming a part of the daily workflow for nurses.

While MOE/MAR has been nothing short of a clinical transformation for nursing at UHN, the transformation is far from over. Nursing Informatics continues to monitor the sustainability of what has been implemented by returning to units on a regular basis to assess nurses' progress and identify additional training needs. Nursing Informatics is also attempting to understand when nurses are taking shortcuts or workarounds, potentially indicating that some elements of the system require further modification.

But, in the final analysis, MOE/MAR has required that nurses interact with the electronic patient record on a continuous basis. As such, nurses are now well positioned for additional electronic patient record applications yet to come.

\section{About the Authors}

Brenda Laurie-Shaw, RN, BScN, MN, is the Director of Nursing Informatics at University Health Network with a cross-appointment with the Faculty of Nursing at the University of Toronto. She is also a founding member of the Ontario Nursing Informatics Group (ONIG) and Canadian Nursing Informatics Association. Contact: brenda.laurie-shaw@uhn.on.ca.

Wendy Taylor, RN, BScN, is the Project Manager, Nursing Informatics - Nursing Lead for Medication Order Entry/ Medication Administration Record and Manager of the Clinical Support Analyst group at University Health Network.

Carol Roach, RN, BScN, MHA, is a Project Manager, Nursing Informatics at University Health Network. 
Commentary

\section{Nursing Perspective: Focus on Clinical Best Practices, Patient Safety and Operational Efficiency}

Lynn M. Nagle

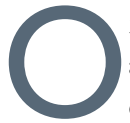

rganizational challenges and struggles with user adoption of computerized clinical applications are common and, as yet, not easily overcome. This case study demonstrates the complexity of the issues and processes that need to be addressed in the implementation of new technologies to support the management of clinical information. These complexities arise in the management of the people, the processes and the technology associated with the change.

More specifically, experiences such as this illustrate that the perceived value and ease-of-use of applications and devices by clinicians are critical to their successful adoption. Healthcare has a pervasive history of information technology solutions being acquired and implemented without considering or rethinking the workflow of users. Although becoming increasingly recognized as a key success factor, the concept of users being engaged and supported as needed - pre- and post-system implementation - remains largely inadequate. Additionally, designating individuals with the responsibility to support the clinicaltechnical translation (e.g., clinical informaticians) is a strategy that has had a meaningful and successful benefit to implementations in many organizations.

The art of redesigning workflow processes to accommodate new tools to manage clinical information is also yet to be mastered by most organizations. Incorporating new applications of technology without rethinking existing practices is likely to result in inefficient and potentially unsafe workarounds. It is also important to consider that new risks and inefficiencies may arise by introducing new technologies into the work environment. Nonetheless, users' mental models and unaffected work processes should also be considered in the overall redesign of specific work activities.

As discovered in the UHN experience, redesigning workflow processes is likely to uncover organizational inconsistencies in practice and the inadequacies in existing policies with respect to new processes. Organizations need to be willing to acknowledge and address the discovery of practices that violate accepted standards and policies. In addition, new strategies may need to be developed to replace lost functions and mitigate new risks (e.g., electronic whiteboard to alert staff to new orders).

The design of applications and technologies to support clinical information management has markedly improved in recent years. However, the verdict is outstanding with respect to the most appropriate device to support the management of clinical information in healthcare settings by nurses and others. Trial and error and the responsiveness and affordability of devices seem to be prevailing criteria in organizations' choices of appropriate solutions. One might speculate that the ideal devices to support clinical computing have yet to be designed. In the meantime, it is unlikely that a single device will be identified to fit with the work patterns in all clinical settings. At best, efforts should be made to assure the device of choice works for the user and can be reasonably integrated with workflow processes without creating more work.

\section{... designating individuals with the respon- sibility to support the clinical-technical translation (e.g., clinical informaticians) is a strategy that has had a meaningful and successful benefit ...}

There is no question that the transition between manual and computerized information management poses many challenges in the delivery of safe clinical care. Similar to the dilemma of determining the most appropriate computing device, the most efficacious order of implementing specific applications remains open to debate. For those in the midst of implementing clinical information systems, early wins can be derived with solutions that are easy to use and bring value-adds to clinicians. This work necessitates thoughtful consideration of the scope and phasing of any implementation. Consider how much risk and disruption your organization is prepared to address in conjunction with the deployment of new technologies. These are disruptive technologies and success is largely dependent upon the diligence, attention and collaborative, collective energy of information technology and clinical experts. Support for users needs to be visible, sustained and grounded in the real world of the clinician community. The need to be accountable for quality, safe care in the face of learning new technologies is a concern for clinicians - a supportive, resource-supplemented infrastructure is essential to continue successful implementation of these new technologies. To chronicle and share experiences such as this is critical to our mutual learning about what works and secures success.

\section{About the Author}

Lynn M. Nagle, RN, PhD, is a Health Informatics Consultant, in Toronto, ON 